ACCEPTED MANUSCRIPT

\title{
Analysis of the optical transmission of a ferrofluid by an electromagnetic mixture law
}

To cite this article before publication: Ángel Sanz Felipe et al 2018 J. Phys. D: Appl. Phys. in press https://doi.org/10.1088/1361-6463/aab05f

\section{Manuscript version: Accepted Manuscript}

Accepted Manuscript is "the version of the article accepted for publication including all changes made as a result of the peer review process, and which may also include the addition to the article by IOP Publishing of a header, an article ID, a cover sheet and/or an 'Accepted

Manuscript' watermark, but excluding any other editing, typesetting or other changes made by IOP Publishing and/or its licensors"

This Accepted Manuscript is @ 2018 IOP Publishing Ltd.

During the embargo period (the 12 month period from the publication of the Version of Record of this article), the Accepted Manuscript is fully protected by copyright and cannot be reused or reposted elsewhere.

As the Version of Record of this article is going to be / has been published on a subscription basis, this Accepted Manuscript is available for reuse under a CC BY-NC-ND 3.0 licence after the 12 month embargo period.

After the embargo period, everyone is permitted to use copy and redistribute this article for non-commercial purposes only, provided that they adhere to all the terms of the licence https://creativecommons.org/licences/by-nc-nd/3.0

Although reasonable endeavours have been taken to obtain all necessary permissions from third parties to include their copyrighted content within this article, their full citation and copyright line may not be present in this Accepted Manuscript version. Before using any content from this article, please refer to the Version of Record on IOPscience once published for full citation and copyright details, as permissions will likely be required. All third party content is fully copyright protected, unless specifically stated otherwise in the figure caption in the Version of Record.

View the article online for updates and enhancements. 


\section{ABSTRACT}

Evolution of the optical transmission of a ferrofluid after magnetic field commutation is analyzed by means of an approach based on the so-called mixture laws: expressions which predict the effective permittivity of heterogeneous media as a function of their constituents' permittivities, their proportions and the way they are arranged. In particular, this work is based on a law proposed by Sihvola and Kong for the effective permittivity of a host substance with ellipsoidal inclusions. Ferrofluids are peculiar examples of this kind of media: with the solvent as host, the inclusions are nanoparticle agglomerates whose shapes become modified by magnetic field exposure. In this work, experimental optical transmission of a ferrofluid is compared with predictions based on Sihvola\&Kong's law. A remarkable coincidence is obtained both in absence of magnetic field, without using any fitting parameter, and in presence of magnetic field, employing the inclusions' average ellipticity as fitting parameter. The results obtained for time dependent optical transmission of a ferrofluid after magnetic field switch on or off allow one to estimate how the average shape of the agglomerates evolves along time. On the other hand, mixture laws are proven to be an interesting alternative to scattering concepts to model the optical transmission changes experienced by ferrofluids once they are exposed to magnetic fields.

Keywords: Ferrofluids, magnetooptics, mixture law, complex permittivity.

\section{INTRODUCTION}

Ferrofluids or magnetic fluids are fascinating substances due to their huge range of commercial and potential applications [1]: rheological and tribological (dynamic sealing, damping, heat dissipation), biomedical (cancer therapy by means of drug targeting or hyperthermia, magnetic separation of cells). The possibility of tuning their optical properties by means of an external magnetic field makes these substances also attractive for photonic applications such as switching, modulation, filtering and all-optical magnetic sensing [2-4]. Some authors have shown experimental results of ferrofluid optical transmission dependence on the applied magnetio field [5-9]. Nevertheless, there is a lack of a model capable of predicting this behavior starting from the characteristics of both the carrier and the nanoparticles. The works devoted to model these behaviors are very scarce and they rely on some fitting parameters to explain the measurements $[10,11]$. Undoubtedly, this is due to the complexity of the phenomena 
involved: Brownian motion combined with magnetic forces that give rise to attraction of nanoparticles and coalescence of chains $[12,13]$, which leads to a distribution of aggregates of different shapes whose detailed interaction with light constitutes a demanding scattering problem [14-16].

The aim of this work consists of proposing an alternative way of explaining the optical transmission in ferrofluids exposed to a magnetic field, not based on scattering concepts but on the so-called mixture laws. This term often refers to formulae which predict the effective dielectric constant or refraction index of a mixture of several homogeneous media, as a function of the characteristic parameters of each component, their proportions, the way they are arranged, etc. [17-26]. Most of these laws, although originally intended for the treatment of lossless constituents, also apply for lossy media as the spectral function formalism makes clear [27-28]: this function, exclusively dependent on the geometrical distribution of the constituents within the composite, determines any effective property concerning any magnitude governed by Laplace's equation.

A good deal of solutions with different ranges of validity has been proposed for mixtures composed of a host medium with sub-wavelength-size inclusions of another medium [20-26]. Specifically, Sihvola and Kong propose a mixture law suitable for heterogeneous media consisting of a host with ellipsoidal inclusions [25]. This approach is interesting for ferrofluids, as they contain agglomerates whose shape is modified in presence of a magnetic field. In absence of it, particles or small agglomerates can be considered spherical in average. If a magnetic field is applied, the agglomerates, apart from increasing in size, become slender. As an approximation, agglomerates can be treated as spheroids, with the polar axis parallel to the magnetic field direction and longer than the equatorial diameter (prolate spheroid). So, as the magnetic field is increased, the proportion between polar axis and equatorial diameter also increases. According to [25], the variation in the shape of the inclusion leads to a change in the effective permittivity and, therefore, to a modification of the optical transmission of the mixture. In this paper, measurements of ferrofluid optical transmission time evolution after magnetic field switch on and off are analyzed by means of the mentioned mixture law. Comparison of transmissions obtained with the magnetic field oriented parallel and perpendicular to the light beam shows a remarkable match with Sihvola\&Kong's predictions [25]. So, this law may constitute an adequate tool to model how the optical transmission of ferrofluids depends on magnetic field. In addition, the good performance of the law proposed in Ref. [25] for these substances allows one to estimate the average aspect ratio of agglomerates by comparing the optical transmission of the mixture at any working condition with its transmission in absence of magnetic field. This way, it is even possible to track how the shape of agglomerates evolves along time.

The paper is organized as follows. Section II is devoted to revise the mixture law proposed in Ref. [25]. Section III presents the experimental setup and contains two subsections: the first one shows the mixture law capability of predicting the ferrofluid transmission in absence of magnetic field and the second one presents the results with applied magnetic field. Section IV presents the conclusions. 


\section{MIXTURE LAW}

The mixture law considered is an adaptation of the Maxwell Garnett formula [17] in order to account for inclusions with ellipsoidal shape and uniform orientation. Here the formula is adapted for spheroids. We refer to the directions as parallel (II) and perpendicular $(\perp)$ with regard to the polar axis of the spheroids. The effective permittivity of a mixture, according to [25], is given by:

$$
\varepsilon_{e f f, U}^{*}=\varepsilon_{e}\left(1+x^{*}\right)
$$

where

$$
x^{*}=\frac{p\left(\varepsilon_{i}^{*}-\varepsilon_{e}\right)}{\varepsilon_{e}+(1-p) N_{U}\left(\varepsilon_{i}^{*}-\varepsilon_{e}\right)}
$$

In the former expressions, the asterisk denotes a complex magnitude, $\boldsymbol{\varepsilon}_{\boldsymbol{e f f}, \boldsymbol{U}}^{*}$ is the mixture effective permittivity in the $U$ direction $\left(U=\|\right.$ or $\perp$ ), $\varepsilon_{e}$ and $\varepsilon_{i}^{*}$ are the host and inclusion permittivities, respectively, $p$ is the volume fraction occupied by the inclusions and $N_{U}$ is the spheroid depolarization factor in the $U$ direction. If the polar and equatorial semi-axes are $\boldsymbol{a}_{\|}$ and $\boldsymbol{a}_{\perp}$, respectively, the depolarization factor $\mathrm{N}_{\cup}$ is [25]:

$$
N_{U}=\frac{a_{\|} a_{\perp}^{2}}{2} \int_{0}^{\infty} \frac{d s}{\left(s+a_{\|}^{2}\right)^{1 / 2}\left(s+a_{\perp}^{2}\right)\left(s+a_{U}^{2}\right)}
$$

The depolarization factors of a spheroid satisfy:

$$
N_{\|}+2 N_{\perp}=1
$$

Figure 1 shows the relationship between the spheroid aspect ratio $\boldsymbol{A}=\boldsymbol{a}_{\perp} / \boldsymbol{a}_{\|}$and the depolarization factors. For the sake of clarity, the inset table particularizes this relationship for three significant cases: when the spheroid becomes a sphere, a needle and a disk.

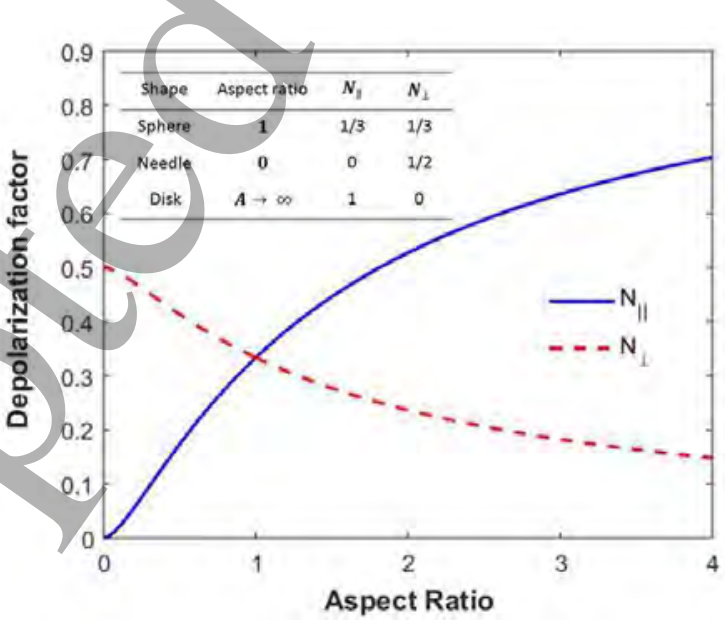

Figure 1. Dependence of the depolarization factors $N_{\|}, N_{\perp}$ on the aspect ratio of the spheroids. The inset table shows the relationship between shape, aspect ratio and depolarization factor in three significant cases. 
As it will be explained next, the direct measurement for characterization is the sample optical transmission factor in the $U$ direction, $T_{U}$. It is well-known that the link between $T_{U}$ and $\varepsilon_{e f f, U}^{*}$ is:

$$
T_{U}=\exp \left(\gamma_{U} L\right)
$$

where

$$
\gamma_{U}=\frac{4 \pi}{\lambda} \operatorname{Im}\left(\sqrt{\varepsilon_{e f f, U}^{*}}\right)
$$

$L$ is the sample width in the light propagation direction and $\lambda$ is the operation wavelength.

\section{EXPERIMENT}

Figure 2 shows the experimental setup employed for determination of the ferrofluid optical transmission as a function of the magnetic field applied. The coils (radius: $16.5 \mathrm{~cm}$ ), are connected to a DC power supply and placed in Helmholtz configuration in order to produce a homogeneous magnetic field. In their central point, the maximum magnetic field generated by them is $72 \mathrm{G}$ (at a rate of $32.4 \mathrm{G} / \mathrm{A}$ ). The ferrofluid is contained in an optical glass cuvette, centered with regard to the coils. The cuvette dimensions are $9.5 \mathrm{~mm} \times 36.8 \mathrm{~mm} \times 2 \mathrm{~mm}$ (inner light path). It is illuminated by a perpendicular He-Ne laser beam (wavelength: 632.8 $\mathrm{nm}$; power: $20 \mathrm{~mW}$ ) expanded and collimated. The beam is expanded in order to measure the transmission as an average over a sample section as wide as possible ( $9.5 \mathrm{~mm}$ in diameter). This way, transmission fluctuations are minimized (for a beam too thin, the sample transmission fluctuations become evident when aggregates are present). Transmitted light is collected by a photodetector. The coils can be rotated around an axis perpendicular to the laser beam, so that the magnetic field and the beam may have parallel (as shown in Figure 2) or perpendicular orientations.

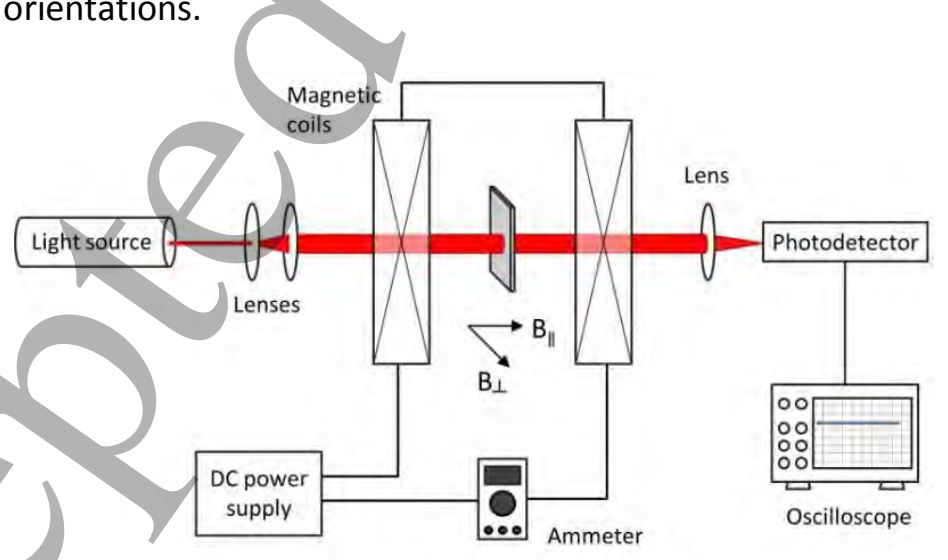

Figure 2. Experimental light transmission setup.

The ferrofluid, supplied by NanoMyP, contains MagP ${ }^{\circledR}$ particles (184 nm of average hydrodynamic diameter, $\mathrm{Pdl}=0.176$ ) suspended in water/SDS as carrier fluid. Figure 3 shows TEM images of the nanoparticles' core of magnetite with a radius of about $50 \mathrm{~nm}$, 
surrounded by the polymeric surfactant that stabilizes the suspended particles in the fluid. The coating composition is 58 wt\% MMA and 42 wt\% EGDMA.
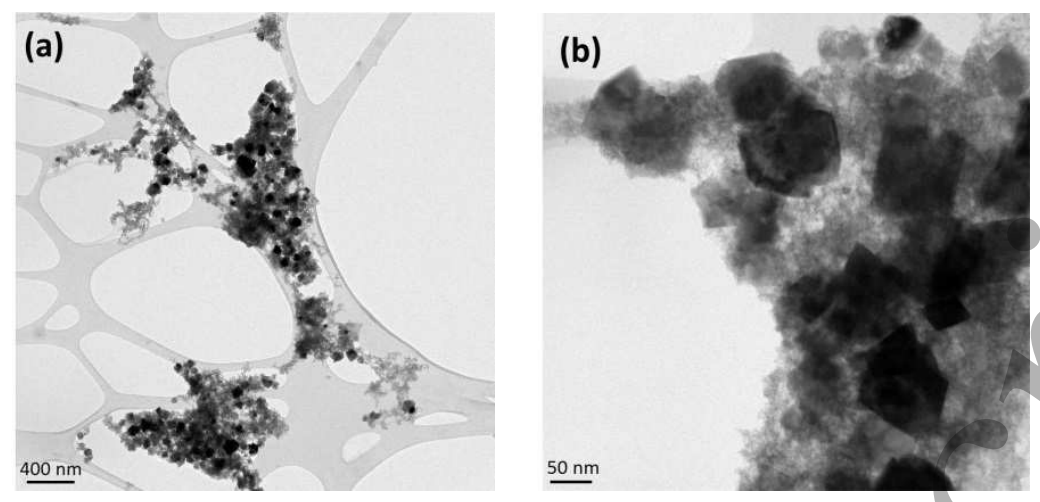

Figure 3. (a)Transmission electron microscope image of the ferrofluid nanoparticles without the carrier fluid for the observation and (b) detail of a group of nanoparticles with radius $\sim 50 \mathrm{~nm}$.

\section{A. Transmission measurements without applied magnetic field}

First of all, the transmissions of the sample with several particle concentrations are measured in absence of applied magnetic field. Figure 4 shows the obtained experimental results, compared with the corresponding theoretical values given by Eqs. (1), (5) and (6), considering the different sample concentrations. For this calculation, obviously $N$ is considered $1 / 3$, as it corresponds to absence of magnetic field, while water and magnetite permittivities considered are, respectively, $\varepsilon_{\boldsymbol{e}}=1.7734$ and $\boldsymbol{\varepsilon}_{\boldsymbol{i}}^{*}=5.5498-0.6947 \mathrm{j}$ [29].

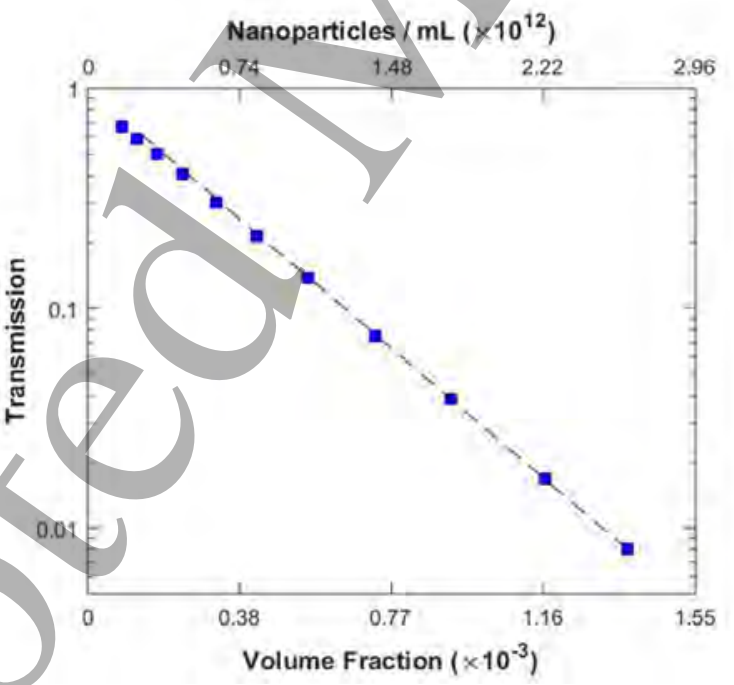

Figure 4. Optical transmission as a function of the volume fraction (bottom) and number of particles/mL (top): experimental results (squares) and mixture law prediction (dashed line).

It can be seen that the agreement is remarkably good. In addition, the theoretical line is not the result of a fitting procedure but a straightforward calculation with the permittivity parameters found in the Literature [29]. So, this result supports the usefulness of the mixture law employed. 
On the other hand, it can be appreciated that the theoretical curve is a straight line. This fact can be explained taking into account that the nanoparticle concentrations employed here yield $\boldsymbol{x}^{*}$ values whose moduli are considerably lower than unity. The maximum modulus corresponds to our maximum concentration, $7.1 \mathrm{mg} / \mathrm{mL}$, which using $\rho_{\text {magnetite }}=5170 \mathrm{~kg} / \mathrm{m}^{3}$ gives $p=1.37 \times 10^{-3}$ and $x^{*}=(1.73-0.18 \mathrm{j}) \times 10^{-3}$. So, it is a good approximation to rewrite $\sqrt{\mathbf{1}+\boldsymbol{x}^{*}} \cong \mathbf{1}+\boldsymbol{x}^{*} / \mathbf{2}$, and consequently Eq. (5) turns to:

$$
\ln T=\frac{4 \pi}{\lambda} L \sqrt{\varepsilon_{e}} \operatorname{Im}\left(1+\frac{x^{*}}{2}\right)
$$

which, considering $1-p \cong 1$, yields:

$$
\ln T=\left\{2 \pi \frac{L}{\lambda} \sqrt{\varepsilon_{e}} \operatorname{Im}\left[\frac{3\left(\varepsilon_{i}^{*}-\varepsilon_{e}\right)}{2 \varepsilon_{e}+\varepsilon_{i}^{*}}\right]\right\} p
$$

This equation shows that the optical transmission represented in logarithmic scale as a function of $p$ (proportional to the nanoparticle concentration) must be a straight line, in good coincidence with the experimental results obtained. It is worthwhile to underline that this expression, valid for low volume fraction of the inclusions in any mixture following Eq. (1), allows one to estimate easily the nanoparticle concentration by means of optical transmission measurements.

\section{B. Transmission measurements with applied magnetic field}

Several series of measurements have been carried out, corresponding to different magnetic field intensities combined with several nanoparticle concentrations and with the parallel or perpendicular orientation of the magnetic field with regard to the laser beam. In each measurement, the time evolution of the optical transmission is registered during $360 \mathrm{~s}$. Magnetic field is switched on at $t=0 \mathrm{~s}$ and it is switched off at $t=180 \mathrm{~s}$.

Figure 5a shows the transmission temporal evolution as a function of the magnetic field intensity (four different magnetic field intensities are considered), both for perpendicular and parallel configurations at a fixed nanoparticle concentration of $7.1 \mathrm{mg} / \mathrm{mL}$. The optical transmission is presented normalized to its value in absence of magnetic field. It is interesting to remark that the curves after magnetic field switch on present some differences in their profiles, that is to say, they do not overlap even though we scale their ordinates. Nevertheless, the curves after switch off show a very similar behavior. Besides, they admit a good fit to an exponential, with a characteristic time around $12 \mathrm{~s}$ in all cases. This similarity in the profile of the different curves suggests a simple Brownian dispersion process without any significant influence of the interaction between nanoparticles once the external magnetic field is switched off. Undoubtedly, the time evolutions observed would present different profiles in ferrofluids with stronger particle-particle interactions, which is a characteristic of certain ferrofluid samples with interest for photonic applications, such as the highly transparent ferrofluids based on cadmium ferrite [30]. 

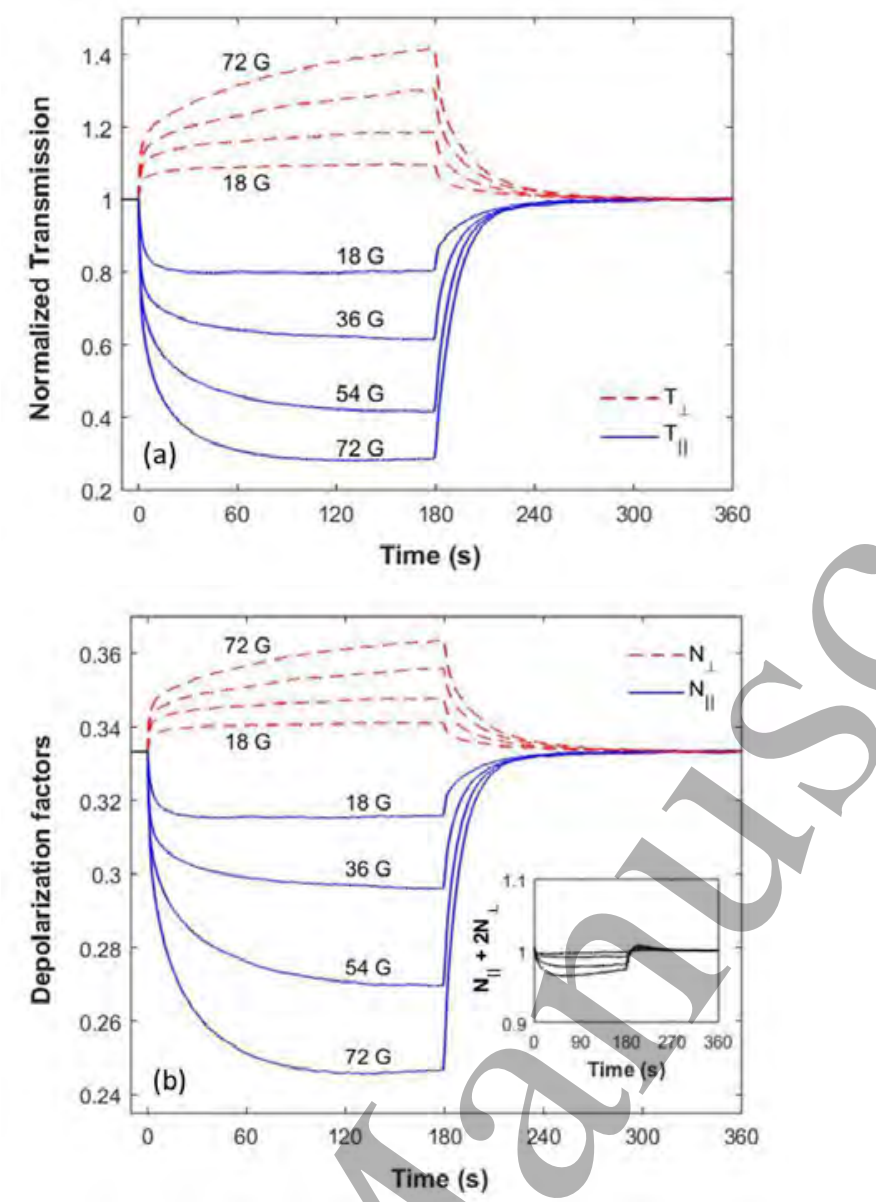

Figure 5. (a) Time evolution of the ferrofluid optical transmission after magnetic field switch on $(t=0 \mathrm{~s})$ and off $(t=180 \mathrm{~s})$, for parallel and perpendicular configurations applying four magnetic field strengths (pointed out close to the curves). (b) Depolarization factors obtained departing from 5.a and the mixture law, Eq. (1). The inset shows the sum $\boldsymbol{N}_{\|}+\mathbf{2} \boldsymbol{N}_{\perp}$ obtained in each case (in order to know which curve of the inset corresponds to which field intensity: the greater the curve deviation from unity, the greater the magnetic field intensity).

According to Eqs. (5) and (6), changes in the transmission require changes in the complex permittivity of the mixture. Their components and proportions are not modified, so that the variations observed must be associated to variations in the depolarization factors or, linked with them, to variations in the aspect ratio of the inclusions. These variations are in agreement with the way the nanoparticles evolve when an applied magnetic field is present. First of all, it must be cleared up that, as nanoparticles coalesce under this condition, the concept of inclusion changes slightly: without coalescence, the term inclusion refers to single nanoparticles; with coalescence, each aggregate can be considered an inclusion. And second of all, these aggregates tend to lengthen along the direction of the magnetic field. Of course they may take different irregular shapes more or less alike to spheroids, but a global change in depolarization factor can be interpreted as an evidence of change in the average aspect ratio of the inclusions. It is straightforward to relate the transmission values with $\mathrm{N}_{\cup}$ through Eqs. (1), (2), (5) and (6). Figure $5 b$ shows the results of Figure 5 a but presented in terms of $N_{U}$. Results show a good agreement with Eq. (4), as the inset emphasizes. It has to be underlined that neither the inclusions are spheroids nor their aspect ratios are homogeneous, so it should 
not be expected that the experimental sum $N_{\|}+\mathbf{2} N_{\perp}$ equals unity. However, the results obtained for this sum are significantly close to one. The comparison between Eq. (4) and the $N_{\|}, N_{\perp}$ values obtained, at any time instant, constitutes a demanding test for the validity of the mixture law in the presence of magnetic field. The good match obtained constitutes a significant support for the validity of Sihvola\&Kong's mixture law for ferrofluids [25], in presence of magnetic field. Besides, this law provides a physical explanation of the trends observed in the transmission changes: the reason why transmission decreases in parallel configuration and increases in the perpendicular one can be explained by relating the modification in the shape of the aggregates with the changes due to this fact in the ferrofluid effective complex permittivity.

According to Eq. (3) and Figure 1, there is a direct link between depolarization factor and aspect ratio. This fact allows one to turn Figure $5 \mathrm{~b}$ results into the aspect ratio values: Figure 6 shows the time evolution of the aspect ratio obtained for the four magnetic intensities considered previously. Some differences in the curve calculation can be observed, depending on whether the depolarization factors considered are $\mathbf{N}_{\|}$or $\mathbf{N}_{\perp}$, as shown in the figure for the $36 \mathrm{G}$ curve. This is a consequence of the imperfect match between their experimental values and Eq. (4), which is justified by the variety of shapes that present the real inclusions, while the mixture law is developed for ellipsoids. The average value between both calculations is shown for the four magnetic field strengths. This figure shows how the aspect ratios decrease as time evolves, which corresponds to an evolution of the aggregate, shapes towards more prolate forms, as expected. Certainly the aspect ratios found here can only be interpreted as effective, average aspect ratios which do not reflect the various aggregate sizes, orientations and shapes that may coexist, but anyway we think that these results provide an interesting insight of the processes of aggregate formation and dissolution.

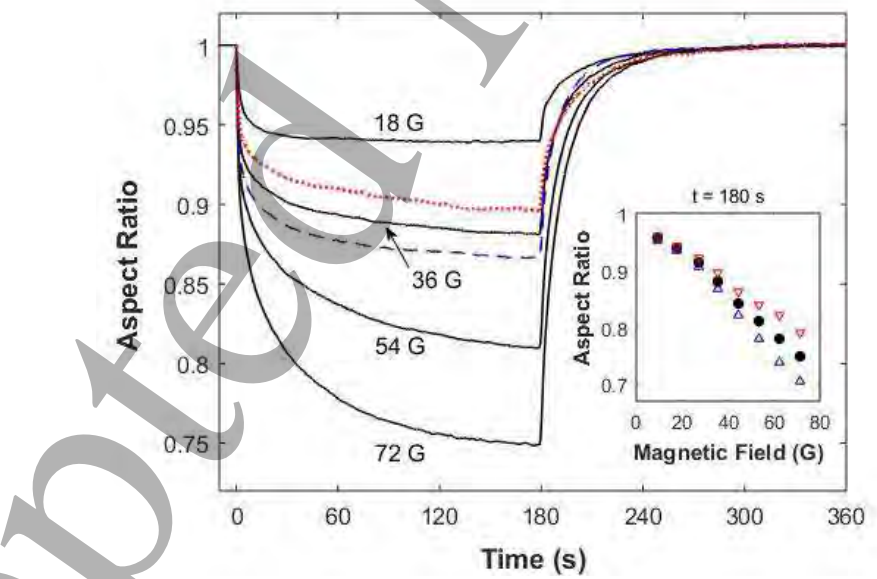

Figure 6. Evolution of the aspect ratios departing from the results of Figure 5 (solid lines). Results obtained from the measurements in parallel (--) and perpendicular (...) configurations are shown just in one case as an example. The inset presents the results obtained at $t=180 \mathrm{~s}$, in which additional measurements at other magnetic field intensities are also included (measurements in parallel: $\Delta$; in perpendicular: V; average: •).

It is also interesting to analyze the influence of the magnetic field intensity on the shape of the aggregates. The inset of Figure 6 summarizes the aspect ratio as a function of the applied 
magnetic field at $\mathrm{t}=180 \mathrm{~s}$. It is clear that the greater the intensity of the magnetic field, the more prolate the aspect ratio obtained, as it could be expected.

Measurements similar to the ones shown in Figure 5a have also been carried out with different nanoparticle concentrations lower than the previous one. The results obtained turn out to be quite similar to Figure 5 but scaled. Figure 7 shows the influence of concentration on the transmission value at $\mathrm{t}=180 \mathrm{~s}$, for different magnetic field intensities applied in parallel configuration (Fig. 7a) and the corresponding aspect ratio according to the mixture law (Fig. 7b). Clearly, the greater the concentration, the more prolate the shape of the aggregate is. This result is also logical taking into account that the ellipticity obtained reflects an average over the inclusions present in the mixture: at very low concentrations aggregates are extremely rare, so most inclusions are individual nanoparticles, whose average shape turns out to be nearly a sphere; as concentration is increased, the proportion of nanoparticles grouped in prolate aggregates also grows and, consequently, the average aspect ratio decreases.
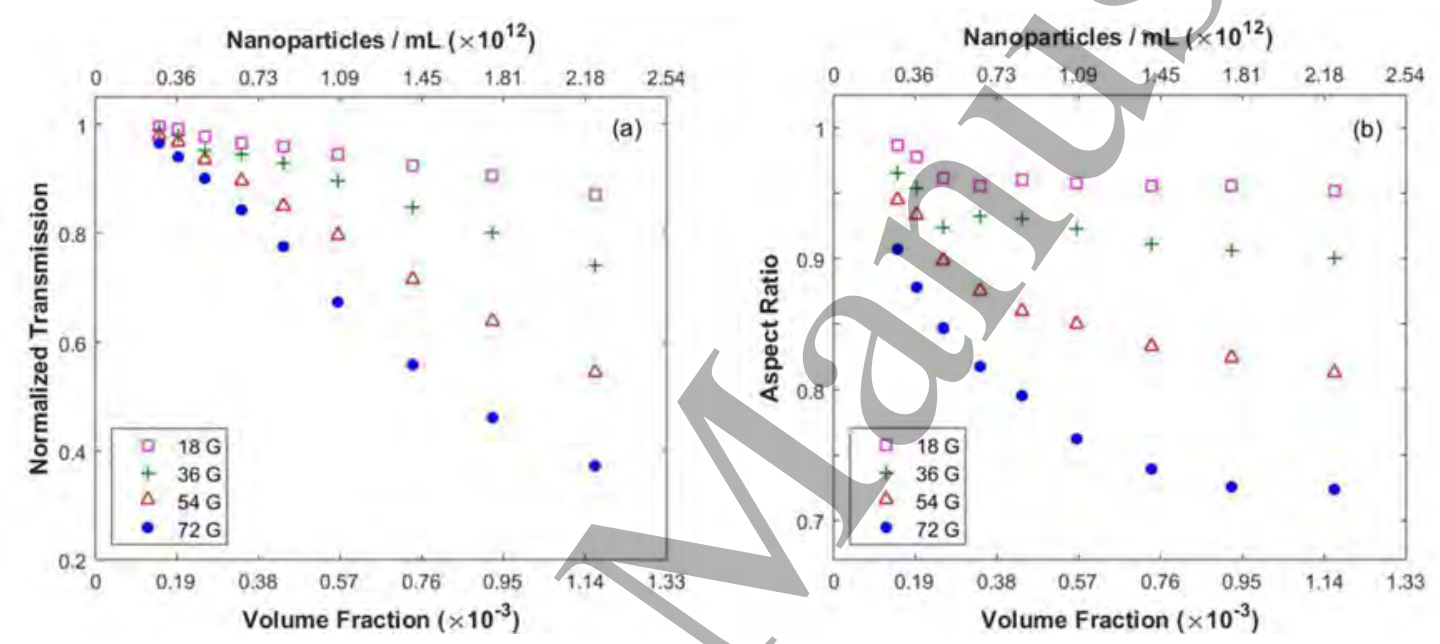

Figure 7. (a) Normalized transmission in parallel configuration at $t=180 \mathrm{~s}$ after magnetic field switch-on, for different volume fractions and magnetic field intensities and (b) corresponding aspect ratios.

\section{CONCLUSIONS}

The mixture law proposed by Sihvola and Kong predicts correctly the optical transmission of a ferrofluid, both in absence and in presence of applied magnetic field. Concerning the good match between theoretical predictions and experiment in absence of magnetic field, it is remarkable that predictions do not involve the fitting of any free parameter, which constitutes a notable success of the mixture law. With regard to the study in presence of magnetic field, we have compared the mixture law predictions with a wide series of measurements obtained by combining several nanoparticle concentrations, different magnetic field intensities and two different orientations of the magnetic field with regard to the light beam (parallel and perpendicular). In every combination, the evolution of the optical transmission after magnetic field switch on and off has been measured. Comparison of each measurement with the mixture formula leads to determination of an average depolarization 
factor. Observation of any pair of results corresponding to perpendicular and parallel transmission of the sample with regard to the magnetic field at the same working conditions shows a good match with the theory, which supports the validity of the mixture law also if the magnetic field is applied. On the other hand, knowledge of the average depolarization factor leads to an estimation of the average aspect ratio of the agglomerates present in the mixture. Based on the link between both magnitudes, it has been possible to estimate the evolution of the average aspect ratio of the agglomerates after magnetic field switch on and off. Its dependence on the applied magnetic field and on the nanoparticle concentrations is also evidenced. The experiment and subsequent analysis presented offer a new approach for tracking the aggregate formation and dissolution processes in ferrofluids. Besides, we prove that effective permittivity is a valid concept to understand optical transmission variations in ferrofluids exposed to magnetic field.

\section{ACKNOWLEDGEMENTS}

This work was supported in part by the national program MINECO TEC2014-52642-C2-2-R and in part by the Universidad de Zaragoza UZ2017-CIE-07.

\section{REFERENCES}

[1] Odenbach S 2002 Ferrofluids: magnetically controllable fluids and their applications (Berlin: Springer)

[2] Pu S, Chen X, Chen Y, Yu Y, Liao W, Chen L and Xia Y 2006 J. Appl. Phys 99093516

[3] Miao Y, Wu J, Lin W, Zhang K, Yuan Y and Song B 2013 Opt. Express 2129914

[4] Miao Y, Ma X, Wu J, Song B, Zhang H, Zhang K, Liu B and Yao J 2015 Opt. Lett. 403905

[5] Brojabasi S, Mahendran V, Lahiri B B and Philip J 2015 Opt. Commun. 342224

[6] Rao G N, Yao Y D, Chen Y L, Wu K T and Chen J W 2005 Phys. Rev. E 72031408

[7] Chen L, Li J, Qiu X, Lin Y, Liu X, Miao H and Fu J 2014 Opt. Commun. 316146

[8] Laskar J M, Philip J and Raj B 2008 Phys. Rev. E 78031404

[9] Patel R, Upadhyay R V and Mehta R V 2006 J. Magn. Magn. Mater. 300 E217

[10] Elmore W C 1941 Phys. Rev. 60593

[11] Eloi M T A, Santos J L, Morais P C and Bakuzis A F 2010 Phys. Rev. E 82021407

[12] Solovyova A Y, Elfimova E A, Ivanov A O and Camp P J 2017 Phys. Rev. E 96052609

[13] Hassan M, Zeeshan A, Majeed A and Ellahi R 2017 J. Magn. Magn. Mater. 44336

[14] Zhang Q, Wang Q J H and Zhu H S 1996 IEEE Trans. Mag. 32297

[15] Yardley J G, McPhedran R C, Nicorovici N A and Botten L C 1999 Phys. Rev. E 606068

[16] Lee S C 2011 J. Opt. Soc. Am. A 281812

[17] Maxwell-Garnett J C 1904 Trans. R. Soc. Can. 203385

[18] Bruggeman D A G 1935 Ann. Phys. 24636

[19] Lichtenecker K and Rother K 1931 Phys. Z. 32255

[20] Hashin Z and Shtrikman S 1962 J. Appl. Phys. 333125

[21] Brosseau C 2006 J. Phys. D: Appl. Phys. 391277

[22] Sihvola A 1999 Electromagnetic mixing formulas and applications (London: IEEE Publishing)

[23] Kärkkäinen K, Sihvola A and Nikoskinen K 2000 IEEE Trans. Geosci. Rem. Sens. 371303

[24] Martín J C, Forniés-Marquina J M and Bottreau A M 2003 Mol. Phys. 1011789

[25] Sihvola A and Kong J A 1988 IEEE Trans. Geosci. Rem. Sens. 26420 
[26] Sihvola A 2013 Phot. Nano. Fund. Appl. 11364

[27] Bergman D J 1988 Phys. Rep. 43377

[28] Milton G W 1981 J. Appl. Phys. 525286

[29] https://refractiveindex.info/

[30] Bazukis A F, Neto K S, Gravina P P, Figueiredo L C and Morais P C 2004 Appl. Phys. Lett. 84 2355 